\title{
Implementation of the Perturbation Methodfor Thermo Elastic Instability in Disk Clutches and Brakes
}

\section{Yun-Bo Yi*}

Department of Mechanical and Materials Engineering, University of Denver, Denver, CO 80208, USA

\begin{abstract}
Thermo Elastic Instability (TEI) is a phenomenon found in high speed rotational devices such as automotive brakes or clutches. When there is frictional heat generation, the interaction between heat transfer and thermal expansion can be unstable, causing high temperature regions on the frictional surfaces. To predict the conditions under which the system yields a rapidly growing temperature field, the perturbation method in conjunction with the finite element scheme has been employed to solve an eigenvalue equation, from which the critical value of the operating speed is determined from the stability boundaries of the leading mode. The method has been found useful for brake and clutch design and optimization in automotive industry.
\end{abstract}

\section{Methodology}

The physical mechanism of TEI was first discovered and explained by Barber in 1969 [1]. Since then a number of analytical and numerical approaches were proposed to solve TEI problems. A direct analytical method is useful for the situations involving simple geometries [2]. However, for complex geometries, a perturbation approach is more preferable. In this method, the temperature is assumed in the form of a steady state solution supplemented with a correction term as follows

$$
T=T_{0}+\mathfrak{R}\left\{e^{b t+j m y} \Theta\right\}
$$

Where $T$ is the temperature, $b$ is the growth rate of temperature and $m$ is the wave number or the hot spot number. $T_{0}$ is the steady state solution. $\Re$ denotes the real part of a complex number. Please note that the above equation can be expressed in both two and three dimensions. This method was first developed for static contact problem, where two thermoelastic bodies at different temperatures are pressed against each other, leading to a heat flux across the contact interface. This alters thermal expansion in both materials and changes the contact area as well [3]. In automotive applications that are related to the prediction of TEI, a similar method has been used with the aid of the finite element method [4]. In this approach we assume a perturbation in the sine or cosine forms and its amplitude grows exponentially in the time domain. The final form of the equation contains a complex eigenvalue that represents the growth rate. The imaginary part of this growth rate is associated with the motion of the "hot spots" relative to the disk circumferentially; whereas the real part is the growth rate itself. The model is divided into a mesh that is biased towards the contact surface so that the temperature gradient at the sliding interface is properly considered. The method has been proved very efficient in determining the unstable mode and the critical speed. It can also predict the coupling between the dynamic and thermoelastic effects in multi-material systems [5].In addition to the finite element approximation, the Fourier reduction method also allows for a significantly efficient solution of the problem in systems with axisymmetric geometries such as annular rings and disks. The method is especially useful for multi-disk clutches, in which the geometry is nearly axisymmetric, as opposed to brakes where the friction pads only cover a small fraction of the rotor.

\section{Results and Conclusions}

It has been found that a transient simulation in the time domain is numerically prohibitive. Using a perturbation method with the Fourier scheme, however, has led to efficient solutions. These solutions have revealed that the leading modes are antisymmetric with respect to the metal disks along the thickness direction, and symmetric with respect to the friction disks. Typically these modes exhibit uniform temperature or focal hot spots around the circumference as shown in Figure 1. Some of the higher order modes also have several waves across the radius as shown in the same figure. Each mode pattern has a unique critical sliding velocity. In automotive brake applications, a dominant hot spot number between six and eight have been obtained. In clutch applications, more hot spots are possibly depending on the geometric and material parameters. It has also been found that the three-dimensional configurations have weak effects on both hot spot number and the associated critical speed for focal modes but they have significant effects on the banding modes where the temperature is constant in the circumferential direction. Finally, the dynamic and thermoelastic effects can be coupled together at low velocities, although the coupling is typically not very strong at speeds above the critical TEI value in the absence of the dynamic effects. Some of the key results have already been confirmed by experiments related to thermal damage in realistic clutch systems. The methods can be used to evaluate the effects
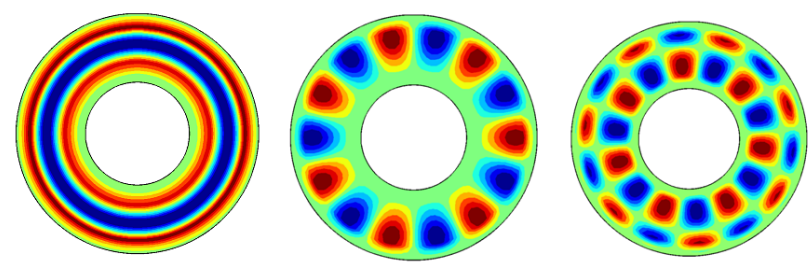

Figure 1: Perturbation analysis revealed multiple TEI modes in disk brakes and clutches.

*Corresponding author: Yun-Bo Yi, Department of Mechanical and Materials Engineering, University of Denver, Denver, CO 80208, USA, Tel: 303-871-2228; E-mail: Yun-Bo.Yi@du.edu

Received January 04 2014; Accepted January 04, 2014; Published January 10 2014

Citation: Yi YB (2014) Implementation of the Perturbation Methodfor Thermo Elastic Instability in Disk Clutches and Brakes. J Appl Mech Eng 3: e126. doi:10.4172/2168-9873.1000e126

Copyright: (c) 2014 Yi YB. This is an open-access article distributed under the terms of the Creative Commons Attribution License, which permits unrestricted use, distribution, and reproduction in any medium, provided the original author and source are credited. 
Citation: Yi YB (2014) Implementation of the Perturbation Methodfor Thermo Elastic Instability in Disk Clutches and Brakes. J Appl Mech Eng 3: e126. doi:10.4172/2168-9873.1000e126

Page 2 of 2

of design changes on the critical velocities of automotive disk brakes and clutches that lead to structural instability.

\section{Future Directions}

Surface separation in clutch operations has been reported in the literature, but it has not been incorporated in this model. Also, the shear stresses due to frictional tractions on the disk surfaces are very important factors leading to vibrations and noise in brakes and clutches, but they are not considered in the current formulation, either. Consequently the present approach does not apply to the dynamic instability directly related to squeal, a phenomenon that is independent of frictional heat generation or thermal expansion. With the dynamic factors incorporated, we expect a better solution to explain and predict brake vibration and noise. In addition, wet clutches are usually immersed in lubricating fluids for the purpose of convective cooling. When lubrication is present, the governing heat transfer and thermoelastic equations need to be altered by introducing some additional terms. As a result different solutions can be expected. For example, the critical sliding velocity will be expressed as a function of the viscosity of the lubricant.

\section{References}

1. Barber JR (1969) Thermoelastic instabilities in the sliding of conforming solids. Proc Roy Soc A. 312: 381-394.

2. Burton RA, Nerlikar V, Kilaparti SR (1973) Thermoelastic instability in a seallike configuration. Wear 24: 177-188.

3. Yeo T, Barber JR (1994) Finite element analysis of the thermoelastic contact stability. ASME J ApplMech 61: 919-922.

4. Yi YB, Barber JR, Zagrodzki P (2000) Eigenvalue solution of thermoelastic instability problems using Fourier reduction. Proc Roy Soc London Series A 456: $2799-2821$.

5. Yi YB (2006) Finite element analysis of thermoelasto dynamic instability involving frictional heating. ASME J Tribology 128: 718-724. 